\title{
Empiema subdural secundario a sinusitis. Descripción de un caso pediátrico
}

\section{Subdural empyema secondary to sinusitis. A pediatric case report}

\author{
A. Herrero, I. San Martín, L. Moreno, M. Herranz, J.C. García, E. Bernaola
}

\section{RESUMEN}

Presentamos el caso de un varón de 9 años derivado al servicio de Urgencias por proptosis ocular derecha y cefalea progresiva en el contexto de una sinusitis, diagnosticado dos días antes por clínica y radiología compatible y en tratamiento con amoxicilina-clavulánico. A la exploración física destaca proptosis derecha con ligera limitación para la mirada conjugada.

Con la sospecha de una posible complicación neurológica de la sinusitis, se realiza tomografía axial computarizada (TAC) craneal, observando empiema subdural frontal derecho. Ingresa para tratamiento antibiótico endovenoso con cefotaxima, vancomicina y metronidazol. Es valorado por los servicios de Neurocirugía, Cirugía Maxilofacial y Otorrinolaringología (ORL) infantil, decidiéndose únicamente drenaje del foco primario, con actitud neuroquirúrgica expectante.

El paciente evoluciona favorablemente con progresiva desaparición de los síntomas. Se realizan resonancias magnéticas periódicas, que muestran clara mejoría hasta resolución completa del empiema. Tras 4 semanas de antibioterapia iv., y tras la normalización clínica y radiológica, el paciente es dado de alta.

Palabras clave. Empiema subdural. Infección bacteriana. Sistema nervioso central. Sinusitis.

\begin{abstract}
We present the case of 9 year old male referred to the A and E service with right ocular proptosis and progressive migraine in the context of a sinusitis diagnosed two days earlier by compatible clinical and radiological tests, and receiving treatment with amoxicillin-clavulanic acid. Physcial exploration revealed right ocular proptosis with a slight limitation for conjugate gaze.

Facing the suspicion of a possible neurological complication of the sinusitis, cranial computer aided tomography (CAT) was carried out, with right frontal subdural empyema observed. He was admitted for intravenous antibiotic treatment with cefotaxime, vancomicin and metronidazole. He was evaluated by child Neurosurgery, Maxillofacial Surgery and Otorhinolaryngology (ORL) services; the decision was taken to only drain the primary focus, while an expectant neurosurgical attitude was maintained.

The patient evolved favourably with a progressive disappearance of the symptoms. Periodical magnetic resonances were carried out, which showed a clear improvement up until the complete resolution of the empyema. Following four weeks of antibiotherapy iv., and after clinical and radiological normalization, the patient was discharged.
\end{abstract}

Key words. Subdural empyema. Bacterial infection. Central nervous system. Sinusitis.
Servicio de Pediatría

Complejo Hospitalario de Navarra

Recepción: 25 de mayo de 2011

Aceptación provisional: 28 de junio de 2011

Aceptación definitiva: 9 de septiembre de 2011

\author{
Correspondencia: \\ Ana Herrero Varas \\ Servicio de Pediatría \\ Complejo Hospitalario de Navarra \\ C/Irunlarrea, 3 \\ Email: aherrerv@navarra.es
}




\section{INTRODUCCIÓN}

El empiema subdural (ESD) es una colección purulenta focal intracraneal localizada entre la duramadre y la aracnoides. La sinusitis es el cuadro predisponente más común, principalmente cuando están afectados los senos frontales, solos o en combinación con los senos etmoidales y maxilares. El ESD también puede surgir como complicación tras invasión directa de microorganismos en el sistema nervioso central (SNC), ya sea por traumatismo craneoencefálico o de manera iatrogénica secundario a neurocirugía o punción lum$\operatorname{bar}^{1,2}$.

El ESD secundario a sinusitis afecta predominantemente a varones (3:1) durante la juventud (20-30 años). Es una complicación poco frecuente, pero potencialmente grave, por lo que precisa una actitud terapéutica precoz y un seguimiento estricto ${ }^{3}$.

Un manejo óptimo de la situación requiere el trabajo conjunto de distintas especialidades, como son Neurocirugía, ORL, Cirugía Maxilofacial y Pediatría.

\section{CASO CLÍNICO}

Varón de 9 años y 8 meses, sin antecedentes de interés, remitido al servicio de Urgencias de Pediatría, desde su centro de salud por cefalea y proptosis. Inicio del cuadro 4 días antes, como fiebre, vómitos, cefalea y congestión nasal; diagnosticado en su centro de salud de sinusitis $\mathrm{y}$ en tratamiento antibiótico con amoxicilinaclavulánico desde hace 48 horas, sin mejoría de los síntomas. Refiere cefalea intensa fronto-temporal derecha y edema en párpado derecho que ha evolucionado hacia proptosis en las últimas horas.

A la exploración física, se evidencia ligera proptosis del ojo derecho, con leve tumefacción palpebral, así como una obstrucción de la fosa nasal del mismo lado. En la exploración neurológica solamente destaca una leve dificultad para la mirada conjugada.

Dada la mala evolución clínica de la sinusitis y la aparición de clínica neurológica, se realiza TAC craneal de urgencia con la sospecha clínica de posible complicación intracraneal. La TAC demuestra una pansinusitis, un flemón subperióstico en pared medial de la órbita derecha y un empiema subdural frontal derecho de pequeño tamaño (1,8 x1,8 x 0,6 cm). (Fig. 1)

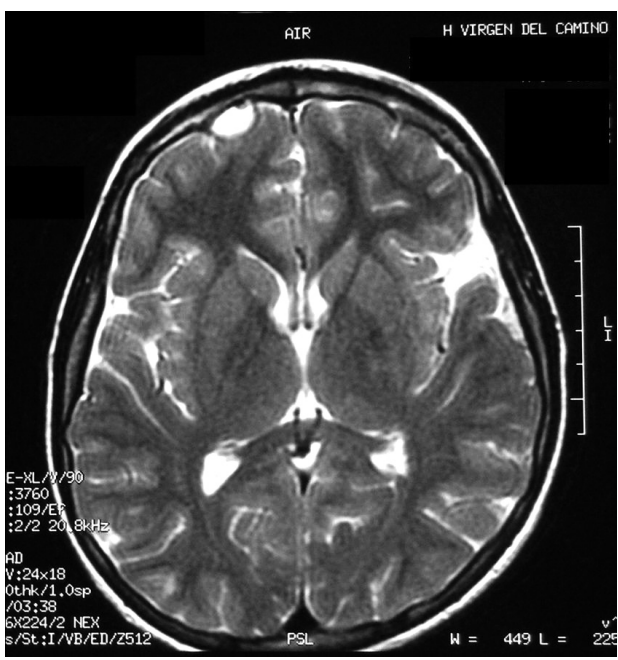

Figura 1. RM con contraste iv (gadolinio) realizada el día del ingreso. Secuencia FSE ponderada en T2. Corte axial a nivel de la porción anteroinferior de la fosa cerebral anterior derecha. Se observa una colección inflamatoria organizada, extra-axial, con dimensiones aproximadas de 1,8 cm.x $0,9 \mathrm{~cm}$. x $2 \mathrm{~cm}$. Su contenido es líquido con un pequeño nivel hidro-aéreo con ligero realce en la periferia.

Valorado de manera conjunta con profesionales del servicio de Neurocirugía, se decide mantener una actitud expectante. Se inicia tratamiento endovenoso empírico con cefotaxima, vancomicina y metronidazol. Así mismo, se realiza drenaje del foco primario (pansinusitis) realizando una evacuación de los senos mediante abordaje nasal. Todos los cultivos resultan negativos.

La evolución clínica es favorable, con desaparición de la sintomatología a los pocos días del inicio del tratamiento. De manera seriada, se van realizando controles de imagen (RM) en los que se aprecia disminución progresiva hasta la desaparición completa a los dieciocho días de tratamiento. Se realiza analítica de sangre en la que se evidencia una normalización de los parámetros infecciosos, con leucopenia e hipertransaminasemia secundarias al tratamiento antibiótico. (Fig. 2)

Se suspende el tratamiento antibiótico a los veintiséis días de su inicio, siendo dado de alta. Posteriormente, es controlado de manera ambulatoria, sin evidenciarse secuelas neurológicas, y con normalización absoluta de los parámetros analíticos. 


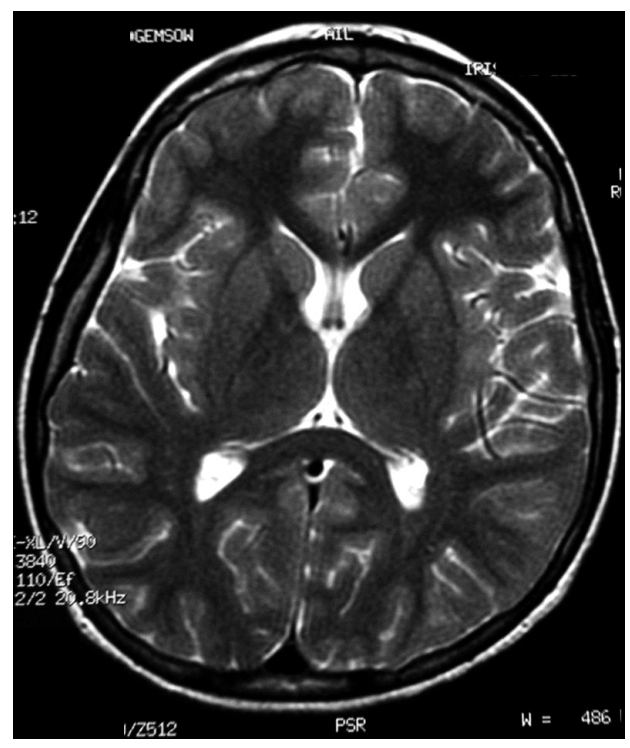

Figura 2. RM con contraste iv (gadolinio) realizada a los 18 días del ingreso. Secuencia FSE ponderada en T2. Corte axial a nivel de la porción antero-inferior de la fosa cerebral anterior derecha. Resolución completa de la colección inflamatoria extra-axial persistiendo un ligero aumento de la captación meníngea a este nivel.

\section{DISCUSIÓN}

EL ESD es una complicación intracraneal de las sinusitis poco frecuente, pero potencialmente grave. En los países occidentales, debido al diagnóstico y tratamiento precoz, aparece con una incidencia aproximada del 1-2\% de los casos de sinusitis. En países subdesarrollados donde el diagnóstico se demora en el tiempo, la incidencia descrita es considerablemente mayor $^{1,2,4}$.

Las complicaciones intracraneales secundarias a sinusitis son, por orden de frecuencia: el absceso epidural, que es a su vez el de mejor pronóstico, el empiema subdural, la meningitis, la encefalitis y el absceso cerebral ${ }^{2}$.

La tríada clínica de fiebre, cefalea y alteraciones neurológicas, propia del diagnóstico de empiema subdural en adultos, sólo aparece en la mitad de los casos pediátricos. La mayoría de los niños presentan, en cambio, una clínica más inespecífica. Casi un 50\% de los casos pueden presentarse con exploración física normal y los síntomas acompañantes más frecuentes en la edad pediátrica son alteraciones oculares, edema facial y hemiparesia ${ }^{1,2}$.

Para su diagnóstico precoz, es importante sospechar una complicación neurológica siempre que la evolución clínica de una sinusitis no es buena.

Siempre que se sospeche una sinusitis complicada es necesario un diagnóstico por imagen (TAC o RM) para descartar complicaciones supurativas intracraneales. En el diagnóstico por imagen, la RM con gadolinio ha demostrado ser superior a la TAC, con una sensibilidad de un $93 \%$ frente a un $63 \%$ para descubrir complicaciones intracraneales. Aunque en muchas ocasiones, por su mayor disponibilidad, se realice inicialmente una TAC para el diagnóstico inicial, es la RM la técnica más sensible y específica para el seguimiento de estos pacientes, además de no producir radiaciones ionizantes y ser por esto más adecuada para el estudio del SNC del niño ${ }^{1,2,5}$.

El ESD es una complicación grave que precisa un tratamiento urgente. Puede llegar a ser letal hasta en un $8 \%$ de los casos pese al tratamiento médico adecuado. Se describen secuelas hasta en un $40 \%$ de los casos, siendo las más frecuentes las paresias y las crisis convulsivas. La aparición de estas secuelas se correlaciona directamente con la duración de los síntomas antes del diagnóstico y con el tiempo transcurrido hasta el inicio del tratamiento ${ }^{2,6}$.

El abordaje terapéutico incluye la instauración precoz de antibioterapia endovenosa y la valoración por los neurocirujanos para considerar una posible intervención. No solo es necesario realizar un abordaje quirúrgico del empiema, sino que en muchas ocasiones es fundamental para la curación el drenaje del foco originario ${ }^{2}$.

El tratamiento antibiótico debe ser endovenoso con una asociación de fármacos que cubran los microorganismos más frecuentes. Como el origen más frecuente del ESD es el sinusal, además de cubrir microorganismos aerobios gram positivos y 
negativos y bacterias anaerobias, no hay que olvidar los microorganismos respiratorios (Estreptococo pneumoniae, Moraxella catharralis y Haemophilus influenza). La duración del tratamiento parenteral dependerá de la evolución clínica del paciente, siendo la duración aconsejada de 2-6 semanas ${ }^{2,5}$.

Como conclusión, destacar que el ESD es una complicación poco frecuente pero potencialmente muy grave, donde es fundamental una sospecha clínica precoz y un adecuado diagnóstico por imagen y que para optimizar el tratamiento del ESD en el niño, es necesario un abordaje multidisciplinar, que incluya la valoración conjunta por parte de los servicios de Neurocirugía, ORL, Cirugía maxilofacial y Pediatría $^{1,2,6}$.

\section{BIBLIOGRAFÍA}

1. Adame, N, Hedlund, G, Byington Cl. Sinogenic intracranial empyema in children. Pediatrics 2005; 116: e461-e467.

2. Germiller Ja, Monin Dl, Sparano Am, Tom Lwc. Intracranial complications of sinusitis in children and adolescents and their outcomes. Arch Otolaryngol Head Neck Surg 2006; 132: 969-976.

3. ONG YK, TAN HK. Suppurative intracranial complications of sinusitis in children. Int $\mathrm{J}$ Pediatr Otorhinolaryngol 2002; 66: 49-54.

4. Passeron H, Sidy Ka A, Diakhate I, Imbert P. Suppurations intracrânienes à porte d'entrée otorhinolaryngologique chez l'enfant au Senegal. Arch Pediatr 2010; 17: 132-140.

5. WALD ER. Acute bacterial sinusitis in children: Clinical features and diagnosis. En: Uptodate, Basow DS (ed), UptoDate, MA, 2001.

6. QuRaishi h, zevallos JP. Subdural empyema as a complication of sinusitis in the pediatric population. Int $\mathrm{J}$ Pediatr Otorhinolaryngol 2006; 70: 1581-1586. 